\title{
Exploring the Usability of Immersive Interactive Storytelling
}

\author{
Jean-Luc Lugrin ${ }^{*}$, Marc Cavazza ${ }^{\dagger}$, David Pizzi ${ }^{\ddagger}$ \\ Teesside University, School of Computing
}

\begin{abstract}
The Entertainment potential of Virtual Reality is yet to be fully realised. In recent years, this potential has been described through the Holodeck ${ }^{\mathrm{TM}}$ metaphor, without however addressing the issue of content creation and gameplay. Recent progress in Interactive Narrative technology makes it possible to envision immersive systems. Yet, little is known about the usability of such systems or which paradigms should be adopted for gameplay and interaction. We report user experiments carried out with a fully immersive Interactive Narrative system based on a CAVE-like system, which explore two interactivity paradigms for user involvement (Actor and Ghost). Our results confirm the potential of immersive Interactive Narratives in terms of performance but also of user acceptance.
\end{abstract}

CR Categories: H.5.1 [Multimedia Information Systems] Artificial, Augmented and Virtual Reality - Virtual Reality for Art and Entertainment.

Keywords: Interactive Storytelling, Virtual Reality, Virtual Actors, Multimodal Interfaces.

\section{Introduction}

The potential of Virtual Reality (VR) to revolutionise digital entertainment has been recognised very early, and has been popularised through the Holodeck ${ }^{\mathrm{TM}}$ metaphor [Murray 1997] which in essence promises spectators to be immersed inside a story world, which would be influenced by their actions. Yet, despite the recent development of Interactive Storytelling as a research field [Foote 2006], there have been very few fully immersive implementations of Interactive Narratives, since this challenge was discussed in one of the VRST 2003 keynotes [Cavazza 2003]. Most of the work in the field of VR has been dedicated to the inclusion of dramatic elements to applications which were primarily in simulation and training [Ponder et al. 2003] [Swartout et al. 2001] as well as being based mostly on single-screen projection systems (one notable exception being AR-Facade [Dow et al. 2007; 2008], which is however an Augmented Reality system).

"e-mail: j-1.lugrin@tees.ac.uk

†e-mail:m.o.cavazza@tees.ac.uk

†e-mail: d.pizzi@tees.ac.uk

§e-mail: thurid.vogt@informatik.uni-augsburg.de

¥e-mail: elisabeth.andre@informatik.uni-augsburg.de

Copyright $\odot 2010$ by the Association for Computing Machinery, Inc.

This is the author's version of the work. It is posted here for your personal use. Not for redistribution. The definitive Version of Record was published in:

VRST 2010, Hong Kong, November 22 - 24, 2010. @ 2010 ACM 978-1-4503-0441-2

\author{
Thurid Vogt ${ }^{\S}$, Elisabeth André ${ }^{\sharp}$ \\ University of Augsburg, Faculty of Applied Informatics
}

In this paper, we explore the baseline usability of immersive Interactive Storytelling, empirically investigating VR as a new entertainment medium [Matsuba et al. 1999]. The rationale for our work is to gain an understanding of the factors that influence user acceptance of an immersive media experience, and of whether the VR reality experience is actually compatible with the interactive storytelling one, in terms of user interaction and story visualisation.

To support this research, we have developed an environment, which integrates all the components of Interactive Storytelling (IS) in an immersive setting (a 4-screens CAVE-like stereoscopic display [Cruz-Neira et al. 1993]). This environment allows users to interact freely with a real-time 3D immersive narrative, based on a dynamic narrative representation, and staged using autonomous virtual characters [Cavazza et al. 2007]. Users can interact with the environment through physical navigation and object manipulation, and with virtual characters through speech recognition, non-verbal behaviour and object-based interaction. We have defined two modes of interaction: in the Actor mode, the user becomes a member of the cast and is expected to play a role, while in the Ghost mode she can navigate freely as an invisible character, still able to interact with the world and the virtual actors. Our objective is to assess whether users are actually able to interact successfully, and in a relevant fashion, with such a complex environment. We also aim to explore possible paradigms for interaction which have not been dedicated sufficient attention in previous interactive narrative research. Finally, we wish to relate objective measures of interaction with elements of subjective user experience.

\section{System Overview}

Our system presents itself as an AI-based Interactive Storytelling system, adapted for stereoscopic immersive visualization and interaction. The IS component follows a character-based storytelling approach: the behaviour of each virtual character is based on a representation of its beliefs and goals, derived from a formalization of its role in the story using an AI formalism for actions (in this case a Planning formalism). The behaviour of virtual actors is driven by a Planning system, which generates a set of narrative actions consistent with the actors' goal. This sequence of actions constitutes the backbone of the narrative. However, actions are generated in real-time, one at a time, and are staged in the virtual environment through virtual actors' physical behaviour. User interactions in the virtual world modify either the world state or the agent's beliefs, so that when the virtual actor generates its next action as part of its role, it propagates the influence of the user intervention potentially altering the course of the story. The background narrative for our system is a classic XIX $^{\text {th }}$ century French novel by Gustave Flaubert, Madame Bovary [Flaubert 1856]. It has been chosen because it emphasizes characters' psychology, with detailed description of the characters' motivation and personalities having been produced by Flaubert as part of the novel's drafts, which have been published [Leclerc 1995]. We have only modelled the actions corresponding 


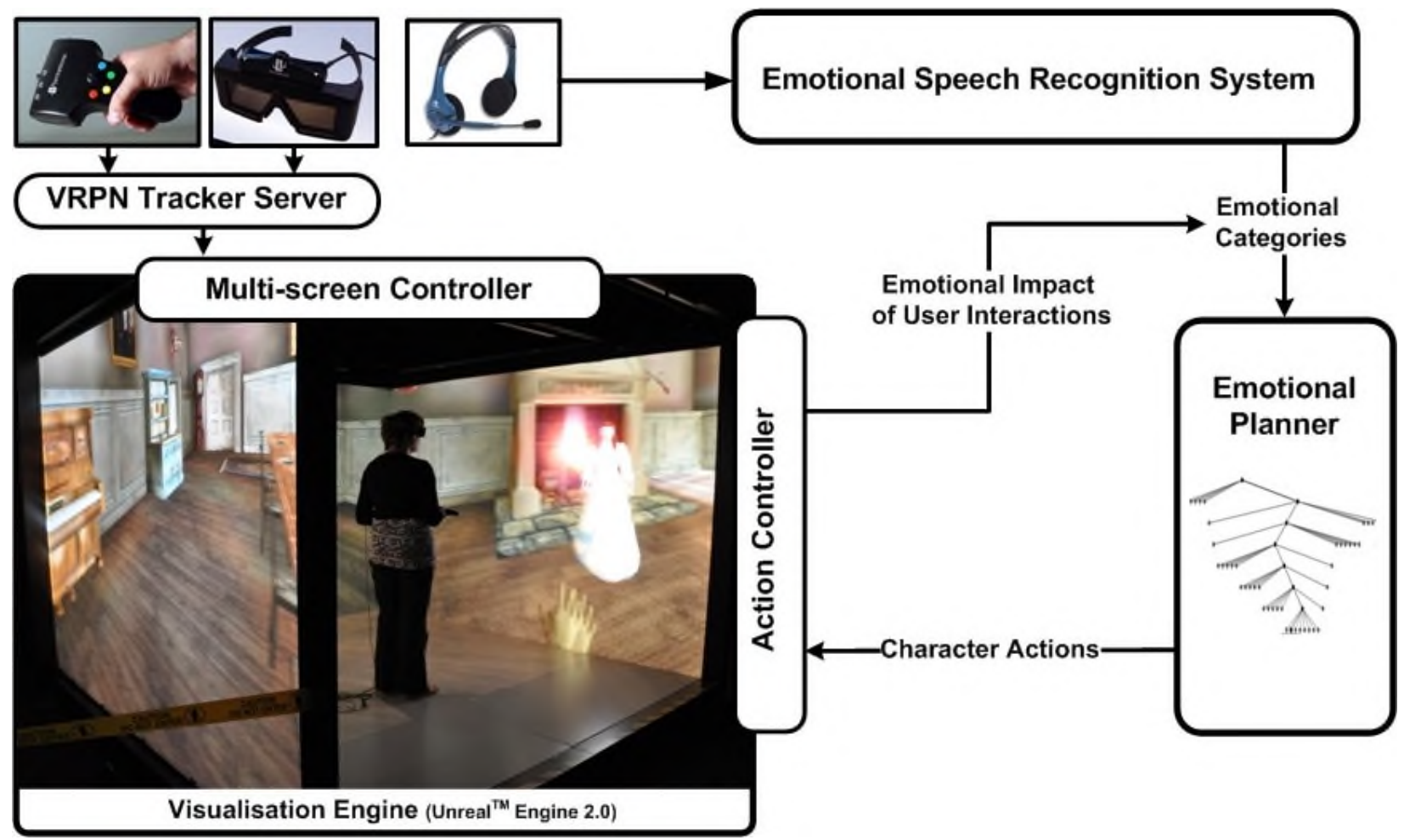

Figure 1: The System is based on a 4-screen CAVE-like display, supporting Multimodal interaction and uses a stereoscopic extension to a game engine

to three chapters $(9-12)$ of the novel, which describe the love affair between Emma Bovary, the wife of a provincial Doctor, and Rodolphe Boulanger, a handsome landowner. The behaviour of Emma and Rodolphe is driven by a representation of their feelings: this representation is used by the Planning system to select which action they undertake, such as offering/refusing a gift, declaring their love, running away as well as deciding on the character's visual behaviour and attitudes.

Because the system is driven by a representation of characters' feelings, interaction modalities are designed to support emotional input as well, which can be used directly to update their beliefs. The system supports multimodal interaction in the form of emotional speech recognition (which allows the extraction of emotional categories from user utterances without placing any constraint on them) and user attitude detection (measuring user distance to a character, along with her body and head orientation to determine attention or interest). Users can interact either by impersonating Rodolphe, in which case Emma will engage in dialogue with them, and their responses (spoken and behavioural) will impact on her emotions, or by trying to influence Emma from outside the play (using again emotional speech recognition). Importantly the users are not informed of this and are told that the system is a speech recognition system, which understands their utterances: we have demonstrated previously [Cavazza et al. 2009] that this supports efficient and believable interaction. Another interaction modality, which naturally derives from the story embodiment in a virtual world, consists in interacting with narrative objects: the user can grab and offer Emma flowers, steal the flowers Rodolphe intended to offer her or the book she wanted to give Rodolphe, all of which has consequences on the emotional state of one or more of the characters.
The emotional model for the characters is based on some of Flaubert's description [Leclerc 1995] and contains 27 feelings, while the emotional categories associated with user interaction, both verbal and non-verbal, are only five (each corresponding to combinations of valence and arousal, see Figure 2): Negative-Passive, Negative-Active, Neutral, Positive-Active and Positive-Passive, and are used to generate transitions between the 29 narrative feelings.

The visualisation of the story as well as the user's interactions with virtual characters and objects are supported by a game engine (Unreal ${ }^{\mathrm{TM}}$ Engine $2.0^{1}$ ), which has been upgraded with a multi-screens controller supporting stereoscopic visualization. The main advantage of using a game engine is to benefit for all the event-based interaction mechanisms between the characters (including the user's avatar where applicable) and the physical environment. Communication between the AI modules controlling characters and the game engine takes place via multiple TCP and UDP sockets, and the overall system works in user real-time for visualization (60 fps) and interaction.

We have adapted the game engine to a four-screens CAVE-like installation of $2.25 \times 2.25$ meters, including real-time head and hand tracking, as well as stereoscopic display at up to 60 frames per second (Figure 1). The real-time tracking in physical space is operated by an Intersense ${ }^{\mathrm{TM}}$ IS900 system $^{2}$, while a VRPN (Virtual Reality Peripheral Network ${ }^{3}$ ) server to handle inputs from the head and wand trackers. Head tracker inputs are used to adjust in real-time the off-axis perspective corrections for each screen, preserving the illusion of depth. This is achieved using a modified OpenGL wrapper library, called $\mathrm{VRGL}^{\mathrm{TM}} 4$, which operates

\footnotetext{
${ }^{1}$ Unreal Engine: http://udn.epicgames.com/Two/WebHome.html

${ }^{2}$ Intersense: http://www.isense.com/products/prec/is 900

${ }^{3}$ VRPN: http://www.cs.unc.edu/Research/vrpn/

${ }^{4}$ VRGL - by Willem de Jonge and PublicVR.
} 


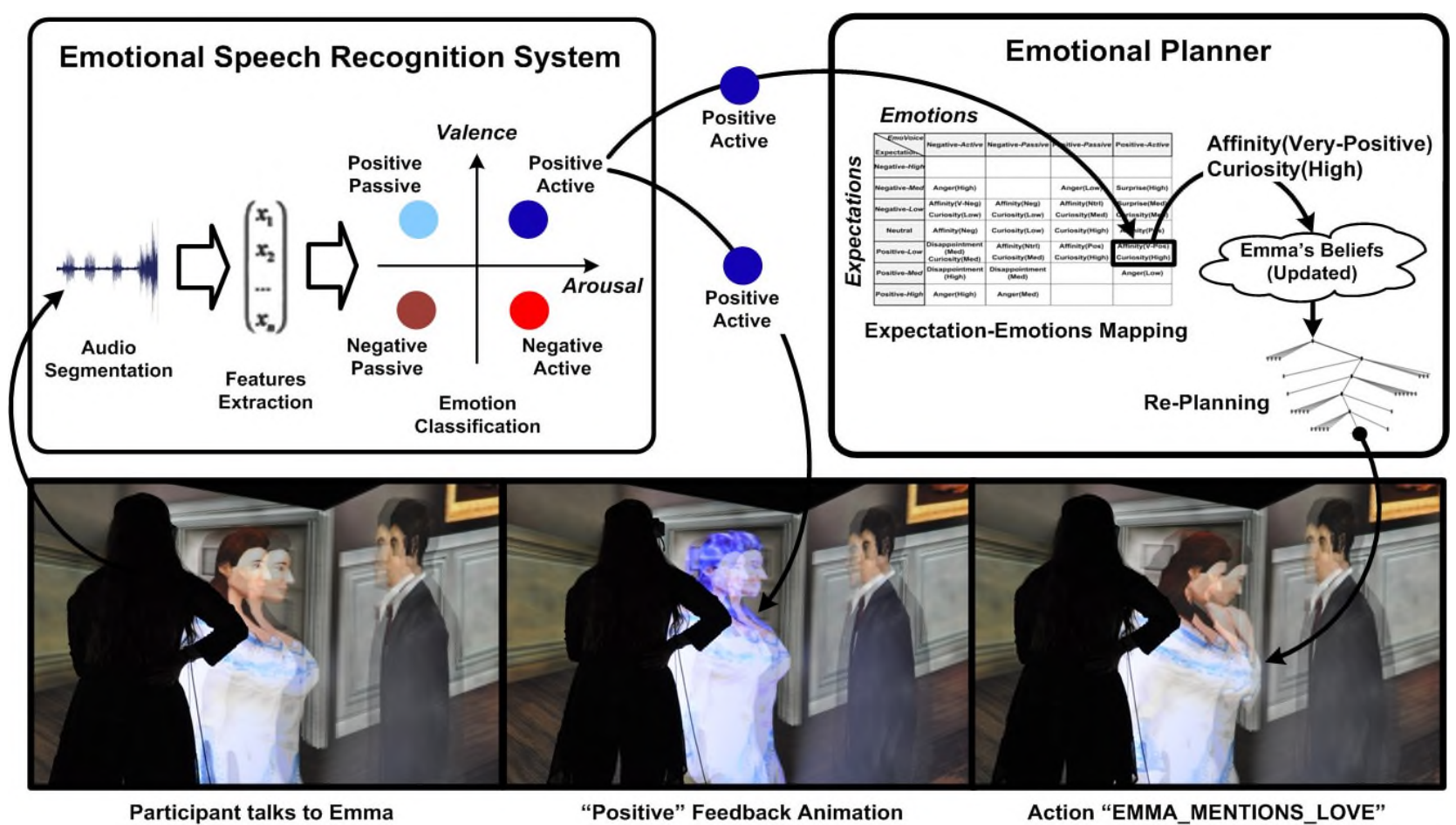

2: Example of Verbal Influences in "Ghost" Mode: the user encourages Emma to pursue her relationship with Rodolphe: "Rodolphe would be perfect for you". This utterance is converted into a positive emotional input. The system gives feedback on the interaction effect by briefly overlaying characters.

between OpenGL and the game engine. As in traditional CAVEs (Figure 1), the user also wears shutter glasses to view the stereoscopic image, which are synchronised with screens rendering using a process known as "Genlocking" handled by the graphics cards (DVG card) of our 4 ORAD $^{5}$ PC cluster. For each screen, one cluster is responsible for mixing both eye view and sends it to a single stereographic projector which will alternate between the left and right eye views at 120 frames per second. In parallel, the wand tracker (Figure 1) is used for both navigation and interactions with objects and characters.

\section{Experimental Settings}

\subsection{Participants}

Thirty-eight users participated in this experiment, including twenty male and eighteen females with an average age of 30.6 years with a range between 19 and 57 years. The average duration of a session was 45 minutes and each subject was rewarded for its participation with a gift voucher equivalent to $24 €$. Participants were instructed to discontinue the experiment at any time in the case of major discomfort.

\subsection{Procedure}

The experiment consisted of five main parts: i) Story introduction ( $\sim 10$ minutes); ii) Practice session ( $\sim 10$ minutes); iii) Experiment sessions A ( $\sim 6$ minutes); iv) Experiment sessions B ( $\sim 6$ minutes),

${ }^{5}$ ORAD - http://www.orad.co.il and finally; v) Completing questionnaires ( $\sim 15$ minutes). During the first part, participants were asked to watch a desktop version of the Interactive Narrative which would demonstrate the type of interaction between characters (both verbal and mediated by objects). Then the two interaction paradigms, Actor mode and Ghost mode, were presented as two separate scenarios, and described as follows to the participants:

- Scenario-A (Actor):"You will be impersonating Rodolphe Boulanger (Emma's lover) where you can address Emma or respond to her complaints and love declaration."

- $\quad$ Scenario-B (Ghost):"You will be invisible but you will still be able to interact (Think of yourself as being a ghost in the middle of a movie scene, capable of whispering to actors, and moving objects around)".

In order to minimise any learning curve effects, the order of these scenarios was andomly chosen for each candidate. However, during this briefing great care was taken not to disclose how user actions may influence the Interactive Narrative. Subjects were offered a practice session to get acquainted with navigation and interaction in a virtual world. During this session, they were gradually introduced to i) navigation controls (i.e. walking and turning), ii) physical interactions mechanisms (i.e. how to grasp, move, throw, or give an object to a virtual character) and iii) speech interaction (i.e. testing emotional speech recognition).

The third part represents the core of the experiment, where users experience Immersive Interactive Storytelling. As discussed in the Results section, for each session user's interactions, navigation, and vision were constantly logged. Finally, participants were requested to complete two questionnaires immediately after their last session. The first one is the Simulator Sickness Questionnaire 


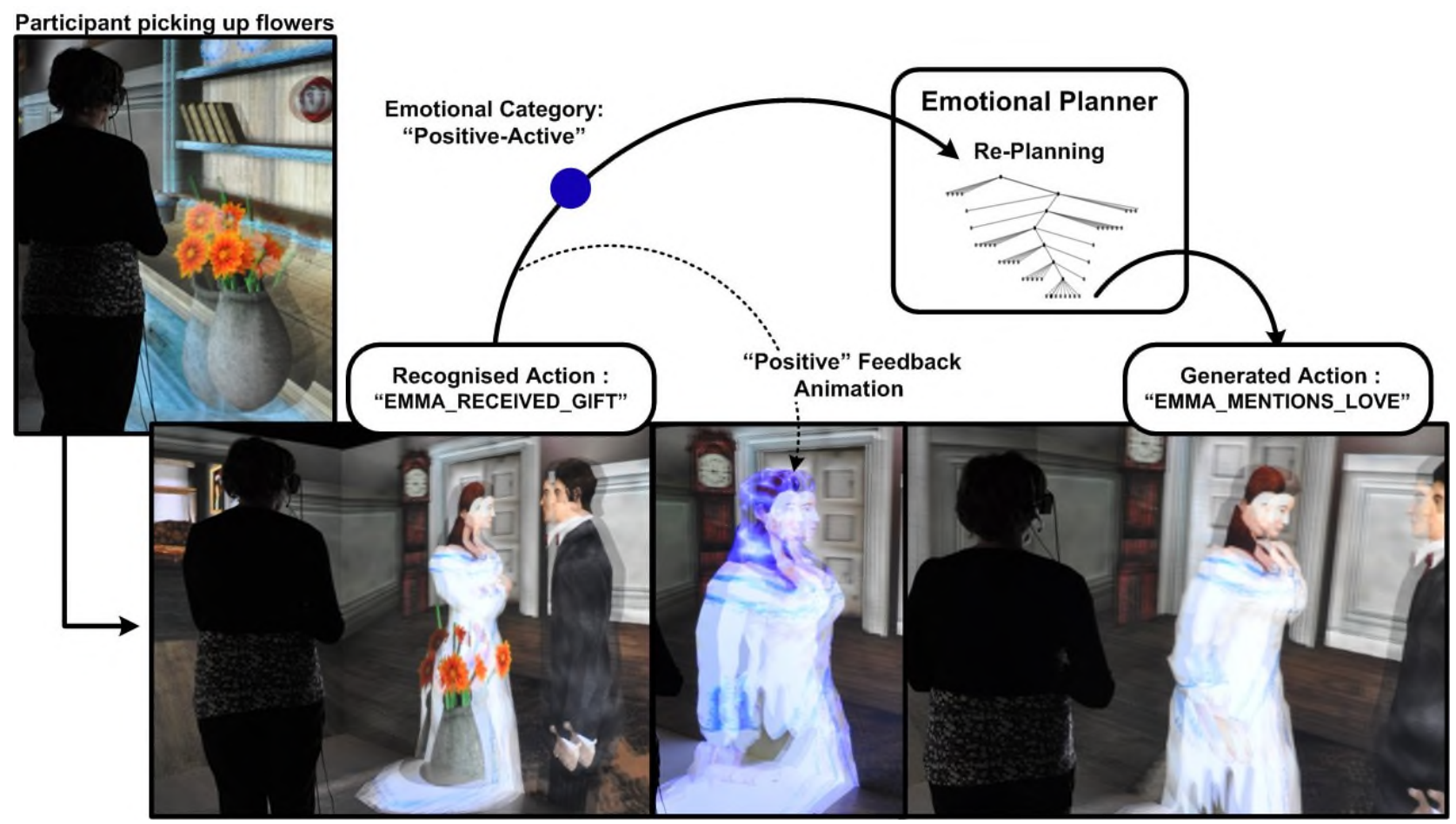

Figure 3: Example of Non-Verbal Influences in "Ghost” Mode: the user gives flowers to Emma, helping Rodolphe to seduce her

(SSQ) developed by Kennedy et al [1993], while the other one is the ITC-SOPI Presence questionnaire proposed by Lessiter et al. [2001]. In addition, participants were free to comment on their experience in a separate form.

\subsection{Environment and Interactions}

As depicted in Figures 1, 2, 3, 4 and 5, the virtual environment reproduces a $\mathrm{XIX}^{\text {th }}$ Century house, with several rooms and a front door patio. The overall size of the environment is about $18 \times 27$ meters, representing an overall walk-able zone of nearly 500 square meters. About 60 reactive objects are distributed throughout the house (flower bouquet, book, lamp, portrait, dish, plate, clock, and piano). Physical interaction with these objects will have an effect on the Interactive Narrative, although the exact effect depends on the current context of the story. As such, these objects have natural semantics that supports their being affordances. However, due to the pacing of the story, which progresses independently of the exploration of the environment by the user, there is a need to facilitate the recognition of such objects within the real-scale environment. This is achieved by using overlay-animated textures, which are triggered when the user comes into proximity of those objects, a solution thus valid for objects of different sizes. For instance, when the user is passing by the piano, animated textures highlight it, suggesting its possible use (see Figure 4). Within the Actor mode, there is only one virtual character present (i.e. Emma Bovary), since the user is playing the part of Rodolphe, whereas both characters appear in the Ghost mode. However, participants were constantly reminded that in both cases they could only interact with (or influence) Emma.

The user navigation model is naturally based on a walking simulation, which has been proven to be intuitive or prominently used in VR [Steinicke et al. 2009] [LaViola et al. 2001] (see
[Usoh et al. 1999] [Bowman et al. 1998] [Zanbaka et al. 2005] for further discussions). The walking speed applied was of 4.15 $\mathrm{Km} / \mathrm{h}$, which correspond to the human average walking speed [Knoblauch et al. $1996(1.22 \mathrm{~m} / \mathrm{s})$ : Banton et al $2005(\approx 1.34 \mathrm{~m} / \mathrm{s})$ ]. In order to adjust walking and rotation speed, we realised a pre-experiment calibration of our navigation mechanism with ten subjects. We observed that a rotation speed between 7-15 deg/s with a constant walking velocity of $1.15 \mathrm{~m} / \mathrm{s}$ produced a satisfying compromise, preventing disorientation while preserving a certain fluidity and naturalness of movement. Furthermore, in order to prevent the impression of gliding instead of walking, our system simulates a "walking oscillation" together with speed-dependent footsteps sound.

Our object interaction technique is a combination of the virtual hand and ray-casting techniques [Milne 1995] used in the PiP system [Lee and Park 2002]. Our virtual hand interaction technique, named "Spring-Hand", is closely inspired by the Go-Go interaction techniques [Poupyrev et al. 1996] and other similar methods to allow at-a-distance manipulation using ray-casting [Bowman and Hodges 1997][Wilkes and Bowman 2008]. A virtual hand is attached to the wand tracker, at a distance equivalent to $25 \mathrm{~cm}$. When the user presses the wand button, the virtual hand extends along the axis pointed by the wand tracker, with a maximum reach distance of two meters and a velocity of $2.5 \mathrm{~m} / \mathrm{s}$. Upon collision with a virtual object, the corresponding effect is automatically triggered depending on the object's semantics. Small artefacts (flowers, books, bottles) tend to be associated a Graspable property, whilst objects possessing a specific behaviour (e.g. producing music for a piano) are Activable (see Figure 4). Therefore, in the case of a Graspable object, the hand brings it back to the user. At this stage, the hand disappears and the user can manipulate the object in a 6-DOF fashion, which is now attached to the wand tracker. The object held can be transferred to one of the virtual agents (mostly Emma) 


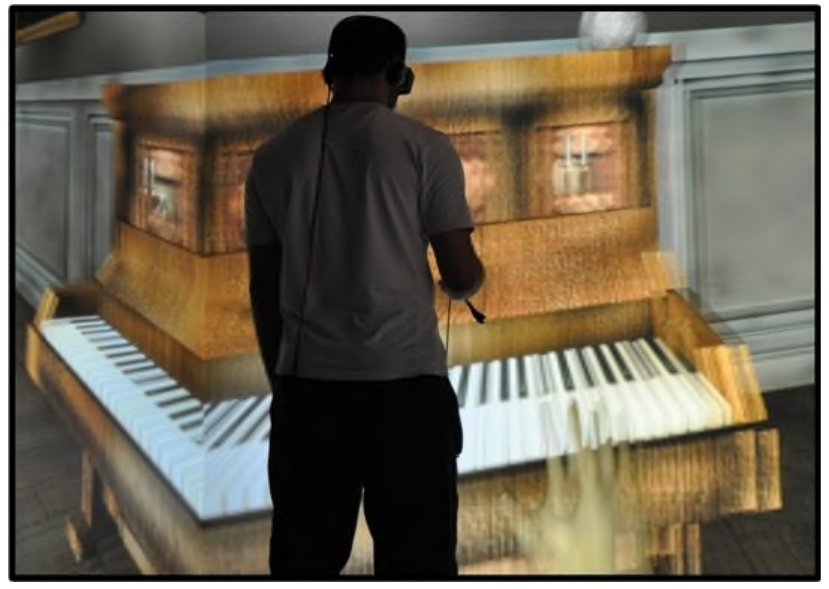

Figure 4: Object activation: a User "playing" piano, which results in a positive emotional input.

simply by holding it and getting it to touch the agent (Figure 3). Likewise, the user can also receive objects from Emma, and decide to accept or decline them by grasping them or not with the virtual hand (Figure 5). The other interaction mode consists in touching objects to activate their intrinsic behaviour. One specific case is constituted by physical contact with Emma, which is interpreted negatively by her. When the hand collides with an object without any semantic properties (e.g. a table, a painting), it immediately bounces back to the user to its default position with no further effect. We have named such events unproductive interactions, as they do not affect the unfolding of the Interactive Narrative. Yet, these are recorded throughout our experiments as they may serve as a measure of interaction attempts and/or exploratory behaviour.

In the Actor interaction mode, user attitudes are detected to be interpreted as non-verbal behaviour. In particular, the perceived attention the user dedicates to Emma can be converted into emotional input. This is a logical consequence of the fact that the user impersonates a character visible to Emma whose attitudes should be part of its communication style. User's attention is measured during dialogue (i.e. when Emma is addressing the user) and is based on distance and orientation (the user should be in close proximity rather than moving to another part of the room and should be facing Emma rather than turning her back on her). This allows to the system to take into account spontaneous user movements supporting again anytime interaction. Emma's response will consist initially in a complaint to the user (Rodolphe) and if the behaviour persists in a direct emotional input equivalent to a Negative-Active emotional input, and a corresponding display of Emma's disappointment.

Speech remains the most natural means of interacting with the characters of a story, especially since the story itself includes dialogue. Speech is compatible with the two main immersive IS paradigms investigated here. In Actor mode, the user will simply participate in the narrative's dialogue, often responding to Emma's lines. In Ghost mode, she will virtually whisper advice to Emma with the aim of influencing her. It should be noted that, in this context, speech interaction is embedded in the interaction paradigms and cannot be used to direct characters or command them to take specific actions. The use of speech in Interactive Storytelling faces a difficult dilemma: speech understanding in the general case is beyond the state-of-the-art, and the type of speech, which should feature in dialogue (e.g. in Actor mode) should be

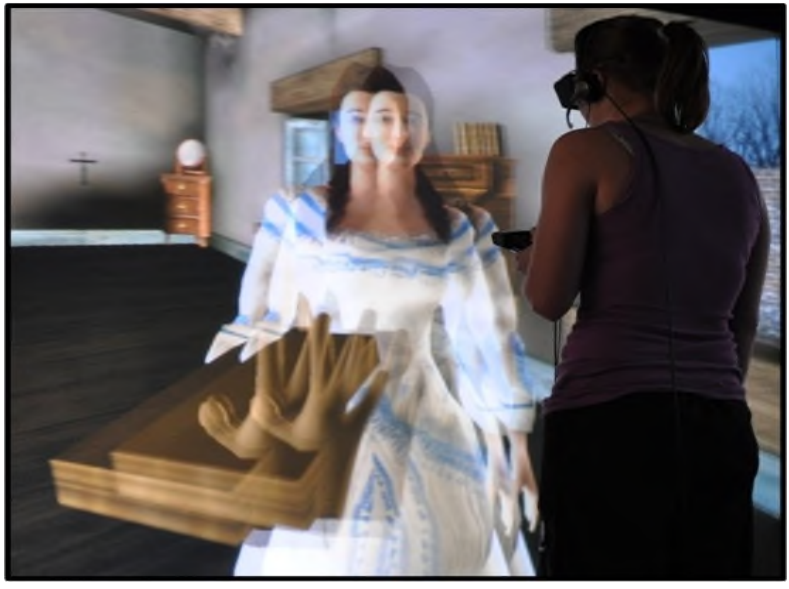

Figure 5: Participant receiving a book from Emma in Actor mode

believable, implying a minimal level of style and sophistication (this being less relevant with the influences in Ghost mode). This dilemma can be solved with the use of emotional speech recognition, which only extracts emotional categories from an utterance, while allowing complete freedom of expression. These will impact into the emotional state of the main character Emma depending on the narrative context. Such an approach allows the user to use speech to interact with Emma without any constraints on style or expressivity: we have shown in a previous study with a desktop version that over $70 \%$ of users believed that Emma actually understood their utterances [Cavazza et al. 2009]. The fact of using the same emotional categories for speech and non-verbal interaction supports multimodal interaction, even though fusion takes place at the level of interpretation by the emotional planner.

\section{Results}

Since the main objective of this study was to explore the usability of Immersive Interactive Storytelling, the most important results are constituted by the objective measures of user interaction during the immersive interactive storytelling experiments (Figures 6-7-8). However, one essential element of user acceptance is also the absence of adverse reactions: this is why we have studied cybersickness for all subjects through the SSQ. Finally, it seemed appropriate to include at least one measure of Presence, although an investigation of the relationship between storytelling and Presence is beyond the scope of this article ${ }^{6}$.

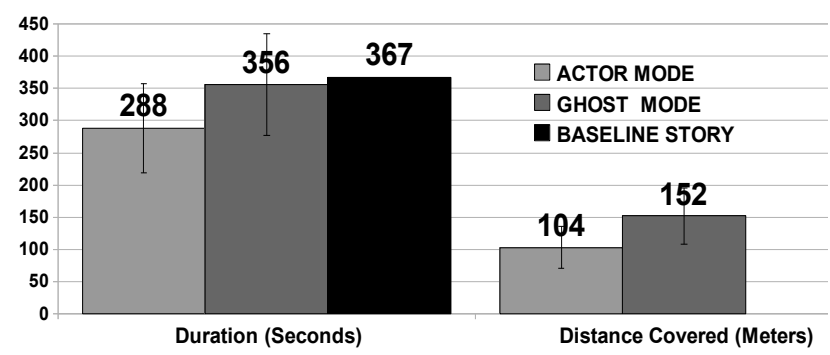

Figure 6: Story Duration and Distance Covered by Users

\footnotetext{
${ }^{6}$ This is also why we have not measured Presence for each IS paradigm but for the experience as a whole.
} 


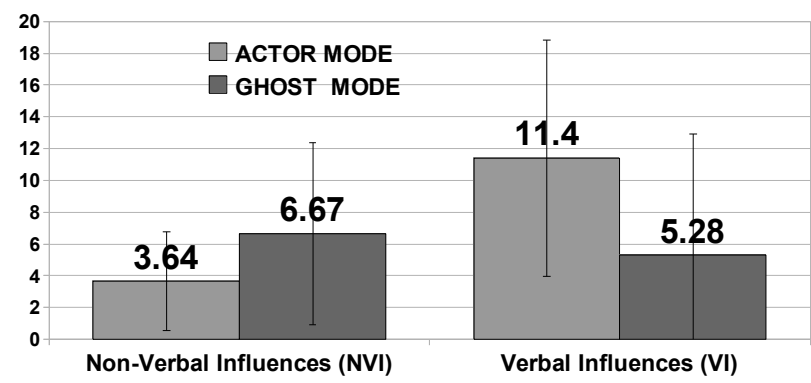

Figure 7: User Interaction Modalities for each Interaction Paradigm

The number of user actions per Interactive Narrative appears largely similar in Actor and Ghost mode ( $\sim 60$ actions), suggesting a comparable level of engagement, regardless of the actual type of the attempted actions. A one-way ANOVA test confirmed a similar engagement level for both modes, by demonstrating that the average number of actions did not differ significantly $(F(145.8)=0.35, p=0.56)$. This is also reflected in the average duration of the Interactive Narrative, between 5 and 6 minutes in total (Figure 6). It is worth commenting on the fact that interactivity shortens the duration of the narrative, as this may appear counter-intuitive, since one would expect that interference would force the autonomous characters to re-plan their behaviour towards new strategies. In reality, eager to interact with the story, users often tend to provide excessive emotional input to the main character Emma, prompting her to accelerate her decisions, leading to an anticipated ending (regardless of the exact ending itself). This only applies to average values, however, and in practice, users have been able to extend the baseline story by 2 minutes or reduce it by nearly 3 minutes in certain cases.

Finally, with an average of $66 \%$ more distance covered in Ghost mode, it appears that users have spent more time exploring the environment (Figure 6). This tendency was clearly evidenced by an analysis of variance showing a significant difference between modes in terms of distance covered $(F(45046.18)=31.37, p$ $<0.001)$. This can have several possible explanations: free exploration of the 3D stage as a way of enjoying the action, search for narrative objects supporting interaction, or simply lack of requirement to stay in close contact with Emma as in the Actor mode. This exploratory tendency is also visible in the high number of unproductive interactions (Figure 8), when users touch non-reactive object with their virtual hand.

Not surprisingly, the ratio of verbal to non-verbal interaction differs significantly in Actor mode $(F(838.51)=14.86, p$ $<0.001)$, where users have to engage in dialogue with Emma as

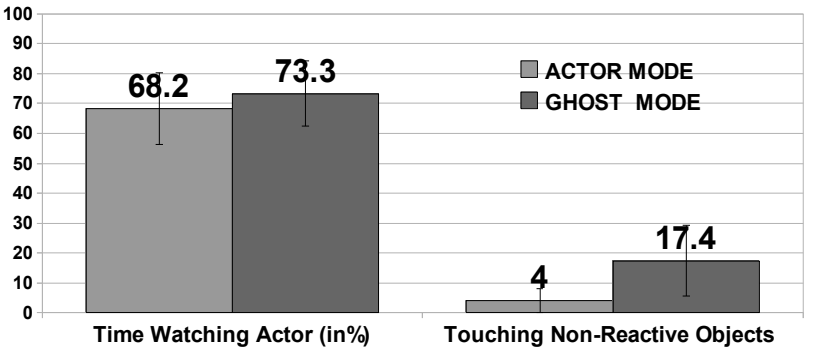

Figure 8: User Attention Metrics

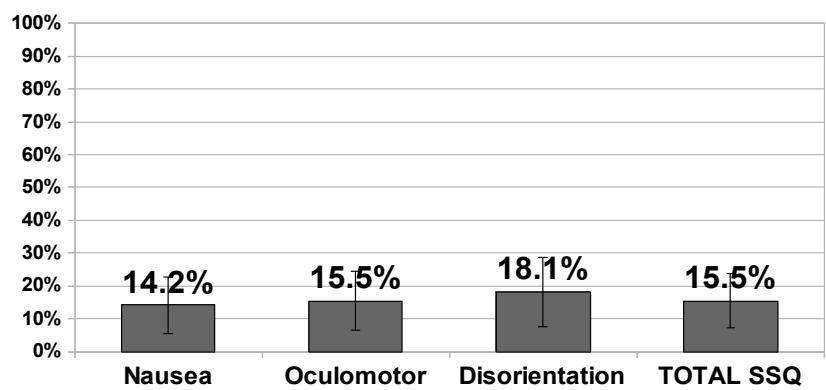

Figure 9: $S S Q$ score results

part of the Interactive Narrative, and in Ghost mode, where there is no such constraint (Figure 7). Non-verbal influences differ in nature between Actor mode (where they are narrative actions such as offering gifts, or non-verbal behaviour during dialogue) and Ghost mode $(F(165.31)=7.87, p<0.001)$, where they can in addition take the form of invisible influences, e.g. stealing narrative objects. For instance, in Ghost Mode, some participants did steal Emma's gift for Rodolphe (a book), and watched the consequences of their intervention, which from a system's perspective, consisted in Emma's "gift" action failing, and, in the story world, resulted in Rodolphe expressing disappointment. It should be noted that users still used verbal interaction in Ghost mode (Figure 7), although its nature was very different from the novel's dialogues. Their intent was still properly captured by the emotional speech recognition system. If we consider the overall duration of the Interactive Narrative, it appeared that while both IS paradigms seem to be equivalent in terms of user involvement (as evidenced by the number of actions), the Ghost mode correspond to longer sessions, probably because of the additional explorations by users (Figure 8 showing more unproductive interactions). Session duration should not be directly interpreted in terms of quality of experience.

Immersive Interactive Storytelling requires prolonged exposure to VR: it is thus necessary to investigate adverse affects such as cybersickness in this context. The exact causes of cybersickness are still debated [LaViola 2000] [Knight and Arns 2006], however several factors have been identified. For instance, latency and jitter has been proven to diminish performance and increase simulator sickness [Steed 2008]. We have minimised such effects firstly by bringing technical improvements to our CAVE-like system in order to considerably reduce the translation and rotation jitters (e.g. by removing native replicated variable aggregation and forcing replications frequencies update for certain data types). As illustrated by Figure 9, the different SSQ scores obtained for each of the cybersickness components, Nausea, Oculomotor and

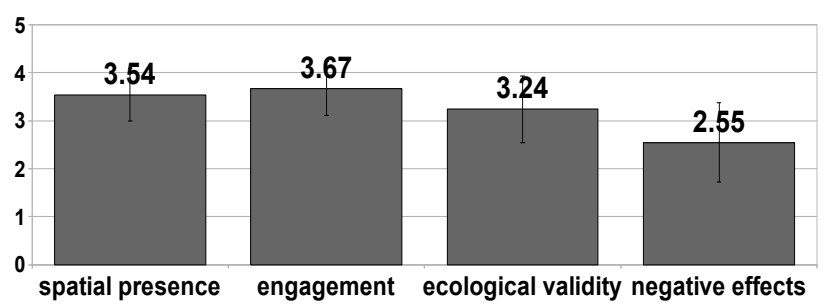

Figure 10: ITC-SOPI score results 
Disorientation are low (around 14-18\%), giving an overall score of $15.5 \%$ for an average exposure time of 22 minutes (two 6-minute immersive narratives and one 10-minute practice session on average). From our 38 participants only one presented severe symptoms (with a Total SSQ Score of $138.38 \approx 60 \%$ ), and has been removed from the study. All the other participants $(>97 \%)$ felt comfortable having completed the whole experiment.

After the experiment, we measured the user self-reported sense of spatial presence and engagement based on the ITC-SOPI Questionnaire [Lessiter et al. 2001] (see Figure 10). The scores analysis confirms a high sense of spatial presence (70\%) as well as important level of user's engagement ( $74 \%$ on average). In their free reports gathered after the experiments, many users have spontaneously made reference to feelings of "being there": " $A$ very good experience, I was surprised about how I felt in that environment. A sense of being there was strong", "I did lose track of time", "I felt immersed", "At a point I felt that I had involuntarily bumped into the table and moved back (even though I know it was not real)". The naturalness of the environment is slightly lower $(64 \%)$ with average score of negative effect $(50 \%)$, which are probably due to the relative simplicity of the environment's graphics and animations, as well as the simplified Physics implemented in the virtual environment to avoid overloading the animation replication system.

$63.2 \%$ of users contributed written comments after the experiments - of these $44.7 \%$ included explicit positive statement such as "I enjoyed the experiment a lot", "I think the experiment was great", "It definitely was an interesting experiment", "Absolutely fantastic- could have done that all day", "I felt that I could really interact with Emma, which made the experience really interesting and pleasurable", "I was able to 'steal' Emma's gift to Rodolphe, thus changing the outcome of the scene, I found it particularly enjoyable". Most of the $21 \%$ comments including negative aspects refer to disorientation; such as "I felt a little disoriented turning around while moving forward" Some comments also expressed certain preferences towards one particular interaction paradigm: "I preferred the role of Rodolphe as I felt there was a definite purpose. As a ghost I didn't feel really involved", "I enjoyed the first part of the story where I took the role of a character as that made me more comfortable in the environment as I had a role".

\section{Conclusions}

These experiments demonstrate that users can successfully engage with an immersive interactive narrative. In particular, if compared with a previous evaluation of the desktop version (for the Actor mode only) [Cavazza et al. 2009], users were able to complete the interactive narrative equally successfully (as confirmed by the average duration of the story), and they did interact frequently, and in a productive fashion, with the interactive narrative. Traditional Interactive Storytelling distinguishes between various levels of user involvement, from the user taking a role, hence being constantly involved (equivalent to our Actor mode), to influencing the story from an external (spectator) position. The latter option, which corresponds to a "lean back" medium, has no equivalent in an immersive context, and has been replaced by our Ghost mode in which the user is in a privileged position to observe the action on stage. This was confirmed by the fact that in ghost mode users still predominantly observed the virtual actors. However, the fact that in ghost mode users kept on interacting and influencing the story (as evidenced by the average story length and number of actions) suggests that such mode can constitute a valid paradigm for Immersive Interactive Storytelling, where it would offer more flexibility in terms of level of involvement. Negative effects and Cybersickness are of course a limiting factor but have only led to interrupting the experiment in exceptional cases $(3 \%)$. It should be noted that negative effects have not been commented by users who successfully completed the story beyond them returning their SSQ. The objective measures of engagement in the interactive narrative are also confirmed by the subjective measures provided by the ITC-SOPI questionnaire (Figure 10). However, despite our interactive narrative being based on affective content (and affective technologies) we have voluntarily left for future work the relations between user emotions and Presence in this context [Freeman et al. 2005] [Hu, and Bartneck 2005].

\section{Acknowledgements}

This work has been funded (in part) by the European Commission under grant agreement IRIS (FP7-ICT-231824).

\section{References}

Banton, T., Stefanucci, J., Durgin, F., Fass, A., And Proffitt, D. 2005. The perception of walking speed in a virtual environment. Presence: Teleoperator Virtual Environments. 14, 4 (Aug. 2005), 394-406.

Bowman, D. A., AND Hodges, L. F. 1997. An evaluation of techniques for grabbing and manipulating remote objects in immersive virtual environments. In Proceedings of the 1997 symposium on Interactive $3 D$ graphics, ACM, Providence, Rhode Island, United States.

Bowman, D. A., Koller, D., And Hodges, L. F. 1997. Travel in Immersive Virtual Environments: An Evaluation of Viewpoint Motion Control Techniques. In Proceedings of the Virtual Reality Annual. International Symposium, 45-52.

Bowman, D. A., Koller, D., And Hodges, L. F. 1998. A Methodology for the Evaluation of Travel Techniques for Immersive Virtual Environments. Virtual Reality: Research, Development, and Applications, 3, 2, 120-131.

CAvazzA, M. 2003. Virtual unreality: storytelling in virtual environments. In Proceedings of the ACM Symposium on Virtual Reality Software and Technology (Osaka, Japan, October 01 - 03, 2003). VRST '03. ACM, New York, NY, 4-6.

Cavazza, M., Lugrin, J., Pizzi, D., And Charles, F. 2007. Madame Bovary on the holodeck: immersive interactive storytelling. In Proceedings of the 15th International Conference on Multimedia (Augsburg, Germany, September 25 - 29, 2007). MULTIMEDIA '07. ACM, New York, NY, 651-660.

Cavazza, M., Pizzi, D., Charles, F., Vogt, T., And André, E. 2009. Emotional input for character-based interactive storytelling. In Proceedings of the 8th International Conference on Autonomous Agents and Multiagent Systems Volume 1 (Budapest, Hungary, May 10 - 15, 2009). International Conference on Autonomous Agents. International Foundation for Autonomous Agents and Multiagent Systems, Richland, SC, 313-320.

Cruz-Neira, C., Sandin, D.J., And DeFanti, T.A. 1993. Surround-Screen Projection-Based Virtual Reality: The Design 
and Implementation of the CAVE. In Proceedings of the ACMSIGGRAPH 1993 Conference, 135-142.

Dow, S., Macintyre, B., AND Mateas, M. 2008. Styles of play in immersive and interactive story: case studies from a gallery installation of AR Façade. In Proceedings of the 2008 international Conference on Advances in Computer Entertainment Technology (Yokohama, Japan, December 03 05, 2008). ACE '08, vol. 352. ACM, New York, NY, 373-380.

Dow, S., Mehta, M., Macintyre, B., and Mateas, M. 2007. AR façade: an augmented reality interactive drama. In Proceedings of the 2007 ACM Symposium on Virtual Reality Software and Technology (Newport Beach, California, November 05 - 07, 2007). S. N. Spencer, Ed. VRST '07. ACM, New York, NY, 215-216.

Flaubert, G. 1856. Madame Bovary. La revue de Paris (Publisher), France, 1856 (in French).

Foote, J. 2006. Where's My Holodeck? The New Frontier of Media Display. IEEE MultiMedia 13, n. 3, 104.

Freeman, J., Lessiter, J., Pugh, K., AND Keogh, E. 2005. When Presence and emotion are related, and when they are not. 8th Annual International Workshop on Presence, September 21-23, London, England.

Hu, J., AND BARTneck, C. 2005. Culture matters - a study on presence in an interactive movie. In Proceedings of the 8th Annual International Workshop on Presence, London, 153-159.

KenNedy, R.S., LANE, N.E., BERBAUM, K.S., AND LiLIENTHAL., M.G. 1993. Simulator sickness questionnaire: an enhanced method for quantifying simulator sickness. International Journal of Aviation Psychology, 3(3), 203-220.

KnIGHT, M. M., AND ARns, L. L. 2006. The relationship among age and other factors on incidence of cybersickness in immersive environment users. In ACM SIGGRAPH 2006 Research Posters (Boston, Massachusetts, July 30 - August 03, 2006). SIGGRAPH '06. ACM, New York, NY, 196.

Knoblauch R.L., Pietrucha M.T., Nitzburg M. 1996. field studies of pedestrian walking speed and start-up time. Transportation research record 1538. Washington: national research council, transportation research board p. 27-38.

LAViola, J. J. 2000. A discussion of cybersickness in virtual environments. SIGCHI Bull. 32, 1 (Jan. 2000), 47-56.

LaViola, J. J., Feliz, D. A., Keefe, D. F., and ZelezniK, R. C. 2001. Hands-free multi-scale navigation in virtual environments. In Proceedings of the 2001 Symposium on interactive 3D Graphics I3D '01. ACM, New York, NY, 9-15.

LeClerC, Y (Ed.). 1995. Plans et Scenarios de Madame Bovary, CNRS Editions, France, (in French).

LeE, G. A., Kim, G. J., AND PARK, C. 2002. Modeling virtual object behavior within virtual environment. In Proceedings of the ACM Symposium on Virtual Reality Software and Technology (Hong Kong, China, November 11 - 13, 2002). VRST '02. ACM, New York, NY, 41-48.

Lessiter, J., Freeman,J., Keogh E., And Davidoff, J. 2001. A Cross-Media Presence Questionnaire: The ITC-Sense of Presence Inventory. Presence: Teleoperators, and Virtual Environments, 10(3): 282-297.
Matsuba, S. N., AND Roehl, B. 1999. Bottom, Thou Art Translated: The Making of VRML Dream. IEEE Computer Graphics and Applications. 19, 2 (Mar. 1999), 45-51.

MiNE, M. 1995. Virtual Environment Interaction Techniques. University of North Carolina Computer Science Technical Report TR95-018.

Murray, J.H. 1997. Hamlet on the Holodeck: The Future of Narrative in Cyberspace, MIT Press, Cambridge.

Ponder, M., Herbelin, B., Molet, T., Schertenlieb, S., Ulicny, B., Papagiannakis, G., Magnenat-Thalmann, N., and THALMANN, D. 2003. Immersive VR decision training: telling interactive stories featuring advanced virtual human simulation technologies. In Proceedings of the Workshop on Virtual Environments 2003 (Zurich, Switzerland, May 22 - 23, 2003). EGVE '03, vol. 39. ACM, New York, NY, 97-106.

Poupyrev, I., Billinghurst, M., Weghorst, S., AND IchiKaWA, T. 1996. The Go-Go interaction technique: Non-linear mapping for direct manipulation in VR. In Proceedings of the ACM Symposium on User Interface Software and Technology, 79.

Riecke, B. E., Schulte-Pelkum, J., AvraAmides, M. N., Heyde, M. V., AND BÜlthoff, H. H. 2006. Cognitive factors can influence self-motion perception (vection) in virtual reality. ACM Transaction on Applied Perception, 3, 3, 194-216.

STEED, A. 2008. A simple method for estimating the latency of interactive, real-time graphics simulations. In Proceedings of the 2008 ACM Symposium on Virtual Reality Software and Technology (Bordeaux, France, October 27 - 29, 2008). VRST '08. ACM, New York, NY, 123-129.

Steinicke, F., Bruder, G., Hinrichs, K., Lappe, M., Ries, B., AND INTERRANTE, V. 2009. Transitional environments enhance distance perception in immersive virtual reality systems. In Proceedings of the 6th Symposium on Applied Perception in Graphics and Visualization (Chania, Crete, Greece, September 30 - October 02, 2009). S. Spencer, Ed. APGV '09. ACM, New York, NY, 19-26.

Swartout, W., Hill, R., Gratch, J., Johnson, W.L., Kyriakakis,C., LaBore, C.,Lindheim, R., Marsella, S., Miraglia, D., Moore, B., Morie, J., Rickel, J., ThiebauX, M., Tuch, L.,Whitney, R., Douglas, J. 2001. Toward the Holodeck: Integrating graphics, sound, character and story. In Proceedings of the 5th International Conference on Autonomous Agents (Agents-01), ACM Press, New York.

Usoh, M., Arthur, K., Whitton, M. C., Bastos, R., Steed, A., SlAter, M., AND BROOKS, F. P. 1999. Walking > walking-in-place $>$ flying, in virtual environments. In Proceedings of the 26th Annual Conference on Computer Graphics and interactive Techniques International Conference on Computer Graphics and Interactive Techniques. ACM Press/Addison-Wesley Publishing Co., NY, 359-364.

Wilkes, C., AND Bowman, D. A. 2008. Advantages of velocity-based scaling for distant $3 \mathrm{D}$ manipulation. In Proceedings of the 2008 ACM Symposium on Virtual Reality Software and Technology (Bordeaux, France, October 27 - 29, 2008). VRST '08. ACM, New York, NY, 23-29.

Zanbaka, C., LoK, B., Babu, S., Ulinski, A., And Hodges, L.F. 2005. Comparison of path visualizations and cognitive measures relative to travel technique in a virtual environment. IEEE Transactions on Visualization and Computer Graphics, $11,6,694-705$. 